\title{
Đánh giá sự phù hợp của hệ thống phân ngành GICS và TRBC: Nghiên cứu thực nghiệm tại Sở Giao dịch chứng khoán Thành phố Hồ Chí Minh
}

\section{Assessment of the suitability of the GICS and TRBC industry classification schemes: A study at Ho Chi Minh stock exchange}

\author{
Lê Khoa Huân ${ }^{1^{*}}$, Trần Thị Ngọc Quỳnh ${ }^{2}$, Lê Đình Nghi ${ }^{3}$ \\ 1,2,3 Trường Đại học Sài Gòn, Việt Nam \\ "Tác giả liên hệ, Email: lekhoahuan@sgu.edu.vn
}

\section{THÔNG TIN}

DOI: $10.46223 / \mathrm{HCMCOUJS}$. econ.vi.15.2.243.2020

Ngày nhận: 20/01/2020

Ngày nhận lại: 02/03/2020

Duyệt đăng: 05/03/2020

Tù khóa:

nhóm đồng nhất, phân ngành, suất sinh lợi

Keywords:

homogeneous group,

industry

classification, return

\section{TÓM TẮT}

Nghiên cứu này nhằm đánh giá sự phù hợp của hai hệ thống phân ngành là GICS (Global Industry Classification Standard) và TRBC (Thomson Reuters Business Classification - TRBC) tại TTCK Thành phố Hồ Chí Minh dựa trên phân tích tương quan SSL giữa các cổ phiếu trong cùng ngành và khác ngành. Sử dụng dữ liệu SSL từ 01/01/2014 đến 31/12/2017, đối với SSL theo tháng, kết quả nghiên cứu chỉ ra trong cả hai hệ thống phân ngành GICS và $\mathrm{TRBC}$, sự khác biệt tương quan suất sinh lợi (SSL) trung bình giữa các cổ phiếu trong cùng ngành và khác ngành có ý nghĩa thống kê. Như vậy, nếu xét dựa trên tiêu chí phân chia các cổ phiếu cùng ngành thành các nhóm đồng nhất, cả hai hệ thống phân ngành TRBC và GICS là phù hợp đối với TTCK Thành phố Hồ Chí Minh.

\begin{abstract}
This study aims to assess the suitability of two industry classification schemes, GICS (Global Industry Classification Standard) and TRBC (Thomson Reuters Business Classification TRBC, ) in Ho Chi Minh City Stock Exchange, based on the analysis of average return correlations between within-industry and outside-industry stocks. Using monthly data from Jan 01, 2014 to Dec 31, 2017, in both GICS and TRBC classification schemes, the results show that there are significant differences between withinindustry and outside-industry correlations. Therefore, both GICS and TRBC are suitable to use in Ho Chi Minh City Stock Exchange.
\end{abstract}

\section{Giới thiệu}

Với xu thế hội nhập của Việt Nam vào kinh tế thế giới, ngày 25 tháng 01 năm 2015, Sở Giao dịch chứng khoán Thành phố Hồ Chí Minh (HOSE) đã chọn chuẩn phân ngành toàn cầu 
GICS (Global Industry Classification Standard) làm chuẩn phân ngành cho các công ty niêm yết trên HOSE (Vuong \& Tran, 2017). Mặc dù đã được áp dụng tại Việt Nam, tuy nhiên, cho đến nay nhóm tác giả tìm được rất ít nghiên cứu đánh giá sự phù hợp của hệ thống phân ngành GICS trên TTCK Việt Nam. Một trong các phương pháp đánh giá sự phù hợp của các hệ thống phân ngành trên TTCK là xác định các nhóm đồng nhất (homogeneous groups), cụ thể là phân tích sự khác biệt giữa tương quan trung bình của SSL cổ phiếu các công ty trong cùng ngành và khác ngành. Phương pháp này đã được ứng dụng trong một số nghiên cứu của (Chan, Lakonishok, \& Swaminathan, 2007; Bhojraj, Lee, \& Oler, 2003),... tại một số TTCK thế giới. Tuy nhiên, tại Việt Nam, nhóm tác giả chỉ tìm thấy nghiên cứu của Vuong và Tran (2017) đánh giá sự phù hợp của hệ thống phân ngành toàn cầu (GICS) tại TTCK TP.HCM. Kết quả nghiên cứu chỉ ra chưa đủ cơ sở để chứng minh tính phù hợp của hệ thống phân ngành GICS khi áp dụng trên HOSE.

Hiện nay, có nhiều hệ thống phân ngành đang được sử dụng trên thế giới như SIC (Standard Industry Code), NAICS (North American Industry Classification System), FF (Fama and French), GICS (Global Industry Classification Standard) và TRBC (Thomson Reuters Business Classification). Từ đó, nhiều nghiên cứu khác nhau đã được sử dụng để đánh giá sự phù hợp của các hệ thống phân ngành này tại nhiều thị trường khác nhau trên thế giới như Chan và cộng sự (2007), Phillips và Ormsby (2016), Bhojraj và cộng sự (2003). Tuy nhiên, ngoài nghiên cứu của Vuong và Tran (2017) chỉ ra hệ thống phân ngành GICS chưa thực sự phù hợp tại Việt Nam, nhóm tác giả chưa tìm thấy nghiên cứu nào đánh giá sự phù hợp của các hệ thống phân ngành khác tại TTCK Việt Nam. Ngoài ra, GICS đã được bổ sung thêm ngành thứ 11 là ngành bất động sản (real estate) từ tháng 9/2016 (MSCI, 2016). Như vậy, việc đánh giá lại mức độ phù hợp của chuẩn phân ngành GICS (sau khi bổ sung ngành bất động sản) với số liệu cập nhật hơn cũng như so sánh với các hệ thống phân ngành khác là cần thiết. Vì vậy, nghiên cứu này sẽ thực hiện đánh giá sự phù hợp của hai hệ thống phân ngành phổ biến là GICS và TRBC.

\section{Cơ sở lý thuyết}

GICS được phát triển bởi tổ chức Morgan Stanley Capital International (MSCI) và Standard \& Poor's (S\&P) vào năm 1999. GICS được đưa ra nhằm thiết lập một tiêu chuẩn chung cho việc phân loại các công ty vào các ngành và nhóm ngành có liên quan với nhau. Chuẩn phân ngành GICS được chia làm 4 cấp độ. GICS gồm 11 Lĩnh vực (sectors), cấp 2 gồm 24 Nhóm ngành (industry groups), cấp 3 gồm 69 Ngành (industries) và cấp 4 gồm 158 Tiểu ngành (sub-industries) (MSCI, 2018).

Phân ngành kinh doanh theo Thomson Reuters (Thomson Reuters Business Classification -TRBC) là hệ thống phân ngành được sở hữu và điều hành bởi Thomson Reuters. Chuẩn phân ngành theo TRBC được chia làm 5 cấp độ: cấp độ 1 gồm 10 Lĩnh vực kinh tế (Economic sector), cấp độ 2 gồm 28 Lĩnh vực kinh doanh (Business sector), cấp độ 3 gồm 54 Nhóm ngành công nghiệp (industry groups), cấp độ 4 gồm 136 Ngành công nghiệp (industries) và cấp độ 5 gồm 837 Hoạt động (activities) (Refinitiv, 2019).

Một trong các phương pháp xác định sự phù hợp của các hệ thống phân ngành là xác định các nhóm đồng nhất. Cụ thể hơn, với một thông tin xuất hiện trên TTCK, các công ty cùng ngành có thể sẽ có phản ứng tương tự nhau, trong khi điều này không hoặc ít xảy ra với các công ty khác ngành. Điều này dẫn đến tương quan SSL trung bình giữa các công ty trong cùng ngành sẽ lớn hơn tương quan SSL trung bình giữa các công ty với các công ty thuộc ngành khác. Như vậy, một hệ thống phân ngành được xem là phù hợp nếu chúng phân loại các công 
ty trên TTCK thành các nhóm đồng nhất, nghĩa là tương quan SSL trung bình giữa các công ty trong cùng ngành lớn hơn tương quan SSL trung bình giữa nhóm ngành đó với các ngành khác có ý nghĩa thống kê. Phương pháp này đã được sử dụng trong nhiều nghiên cứu trước đây tại nhiều TTCK khác nhau trên thế giới. Cụ thể Bhojraj và cộng sự (2003) đã so sánh các tiêu chuẩn phân ngành phổ biến là Standard Industrial Classification (SIC), North American Industry Classification System (NAICS), Fama-French (FF) và Global Industry Classification Standard (GICS). Sử dụng dữ liệu của 1500 công ty(S\&P 1500) trong giai đoạn từ 1994 đến 1999, kết quả nghiên cứu chỉ ra tiêu chuẩn GICS phân loại doanh nghiệp tốt hơn dựa trên tiêu chí các nhóm cùng ngành sẽ tương quan chặt chẽ với nhau (nhóm đồng nhất). Chan và cộng sự (2007) sử dụng dữ liệu suất sinh lợi của tất cả các công ty được niêm yết tại Mỹ trong giai đoạn từ năm 1975 đến 2004 để tính toán tương quan trong ngành và ngoài ngành ứng với các hệ thống phân ngành GICS và $\mathrm{FF}$. Kết quả nghiên cứu chỉ ra rằng tương quan suất sinh lợi giữa các cổ phiếu trong ngành lớn hơn tương quan ngoài ngành đối với cả hai hệ thống phân loại ngành ở trên. Tương tự, Hrazdil, Trottier, và Zhang (2013) đã so sánh bốn hệ thống phân loại ngành là GICS, S\&P, NAICS và FF tại thị trường chứng khoán Mỹ. Sử dụng dữ liệu từ tất cả các cổ phiếu tại thị trường NYSE và NASDAQ trong thời gian từ 1990 đến 2009, kết quả nghiên cứu đã chỉ ra hệ thống GICS có nhiều ưu điểm và phù hợp nhất trong các hệ thống phân loại ngành được xem xét. Dựa vào các nghiên cứu trước, để đánh giá sự phù hợp của hai hệ thống phân ngành là GICS và TRBC, nghiên cứu kiểm định các giả thuyết nghiên cứu như sau:

H1: Trong hệ thống phân ngành GICS, không có sụ khác biệt giữa trung bình tương quan SSL trong ngành và ngoài ngành

H2: Trong hệ thống phân ngành TRBC, không có sụ khác biệt giũa trung bình tương quan SSL trong ngành và ngoài ngành

\section{Dữ liệu và phương pháp nghiên cứu}

Dữ liệu nghiên cứu bao gồm giá và dữ liệu phân ngành theo hai tiêu chuẩn GICS và TRBC của tất cả các công ty đang niêm yết trên HOSE từ 01/01/2014 đến 31/12/2017, dữ liệu được lấy từ nguồn dữ liệu Datastream. Các cổ phiếu không có dữ liệu về giá trong suốt giai đoạn nghiên cứu hoặc thiếu dữ liệu về phân ngành sẽ bị loại khỏi mẫu nghiên cứu.

Các công ty trong mẫu được phân chia vào các ngành tương ứng theo hệ thống phân ngành GICS và TRBC. Để tăng độ tin cậy cho kết quả, mỗi ngành phải có ít nhất 3 công ty. Nếu ngành nào không có đủ 3 công ty sẽ không thực hiện tính toán trung bình tương quan SSL. Nghiên cứu này chỉ tập trung phân tích ở phân ngành cấp 1 của cả 2 hệ thống phân ngành.

Dựa trên phương pháp nghiên cứu của Chan và cộng sự (2007), nghiên cứu thực hiện các bước sau:

Tính toán tương quan trong ngành và ngoài ngành: Đặt $K$ là số cổ phiếu trong mẫu. Dựa vào các chuẩn phân ngành GICS và $\mathrm{TRBC}$, nhóm tác giả thực hiện phân loại các công ty vào từng ngành $I$ theo các chuẩn phân ngành này. Giả sử 1 ngành chứa công ty $i$ bao gồm $N$ công ty, tương quan bình quân của SSL theo từng cặp giữa cổ phiếu $i$ và mỗi cổ phiếu khác trong cùng ngành được tính như sau:

$$
\rho_{i}=\frac{\sum_{j \in I, j \neq i} \rho_{i j}}{N-1}
$$


với $\rho_{i j}$ là tương quan giữa SSL giữa cổ phiếu $i$ và $j$, cổ phiếu $i$ và $j$ thuộc ngành $\mathrm{I}$. Tương tự, tương quan bình quân của SSL theo từng cặp giữa cổ phiếu $i$ thuộc ngành $I$ với tất cả các cổ phiếu không thuộc ngành $I$ được tính như sau:

$$
\emptyset_{i}=\frac{\sum_{j \notin I} \rho_{i j}}{K-N}
$$

Tương quan bình quân của SSL tất cả cổ phiếu trong ngành I được tính như sau:

$$
\rho_{I}=\frac{\sum_{i=1}^{N} \rho_{i}}{N}
$$

với $\rho_{i}$ là tương quan bình quân của suất sinh lợi theo từng cặp của cổ phiếu $i$ thuộc ngành $I, N$ là số cổ phiếu trong ngành $I$.

Tương quan bình quân của SSL giữa một cổ phiếu trong ngành $I$ và các cổ phiếu khác không thuộc ngành I được tính như sau:

$$
\emptyset_{I}=\frac{\sum_{i=1}^{N} \emptyset_{i}}{N}
$$

Tương quan bình quân của suất sinh lợi trong ngành tất cả các cổ phiếu trong mẫu được xác định như sau:

$$
\bar{\rho}=\frac{\sum_{i=1}^{K} \rho_{i}}{K}
$$

Tương quan bình quân của suất sinh lợi giữa một cổ phiếu trong mẫu và các cổ phiếu không cùng ngành được xác định như sau:

$$
\bar{\emptyset}=\frac{\sum_{i=1}^{K} \emptyset_{i}}{K}
$$

Ta thấy rằng $\bar{\rho}$ là trung bình có trọng số (trọng số là số công ty trong mỗi ngành) của tương quan bình quân SSL của tất cả các cổ phiếu trong các ngành $\left(\rho_{I}\right)$ và $\bar{\emptyset}$ là trung bình có trọng số (trọng số là số cổ phiếu trong mỗi ngành) của tương quan bình quân SSL giữa một cổ phiếu trong 1 ngành và các cổ phiếu khác không thuộc ngành đó $\left(\emptyset_{I}\right)$. Nghiên cứu đánh giá sự phù hợp của các hệ thống phân ngành thông qua kiểm định sự khác biệt của tương quan trung bình giữa các công ty trong cùng ngành (giá trị $\bar{\rho}$ ) và các tương quan trung bình giữa các công ty khác ngành (giá trị $\bar{\emptyset})$.

\section{Kết quả nghiên cứu}

Nghiên cứu thực hiện phân nhóm các công ty theo các tiêu chuẩn phân ngành GICS và TRBC. Sau đó, nghiên cứu thực hiện tính toán trung bình tương quan SSL của các cổ phiếu trong cùng ngành và trung bình tương quan SSL cổ phiếu khác ngành ứng với từng phân ngành cấp 1 của cả hai hệ thống phân ngành là GICS và TRBC. Các bước trên được thực hiện thông qua lập trình trên phần mềm Matlab 2016, kết quả nghiên cứu được trình bày trong bảng sau: 


\section{Bảng 1}

Tương quan SSL theo ngày trong ngành và ngoài ngành của hệ thống phân ngành GICS

\begin{tabular}{|l|c|c|c|c|}
\hline Phân ngành theo GICS & Trong ngành & Ngoài ngành & Khác biệt & $\begin{array}{c}\text { Số Công ty } \\
\text { (Tổng =276) }\end{array}$ \\
\hline Năng lượng (10) & 0.207 & 0.118 & 0.089 & 9 \\
\hline Nguyên vật liệu (15) & 0.074 & 0.078 & -0.005 & 46 \\
\hline Công nghiệp (20) & 0.088 & 0.084 & 0.003 & 76 \\
\hline HTD không thiết yếu (25) & 0.048 & 0.062 & -0.014 & 29 \\
\hline HTD thiết yếu (30) & 0.057 & 0.068 & -0.011 & 30 \\
\hline Chăm sóc sức khoẻ (35) & 0.085 & 0.069 & 0.016 & 9 \\
\hline Tài chính (40) & 0.235 & 0.122 & 0.113 & 19 \\
\hline Công nghệ thông tin (45) & 0.050 & 0.069 & -0.019 & 6 \\
\hline Dịch vụ tiện ích (55) & 0.062 & 0.067 & -0.005 & 18 \\
\hline Bất động sản (60) & 0.140 & 0.100 & 0.040 & 34 \\
\hline Trung bình đơn giản & $\mathbf{0 . 1 0 4}$ & $\mathbf{0 . 0 8 4}$ & $\mathbf{0 . 0 2 1}$ & \\
\hline Trung bình có trọng số & $\mathbf{0 . 0 9 6}$ & $\mathbf{0 . 0 8 3}$ & $\mathbf{0 . 0 1 3}$ & \\
\hline t-statistic & & & 1.427 & \\
\hline p-value & & & 0.187 & \\
\hline
\end{tabular}

Nguồn: Tính toán của nhóm tác giả dựa trên Matlab 2016

Kết quả nghiên cứu cho hệ thống phân ngành GICS chỉ ra tại TTCK Thành phố Hồ Chí Minh, trong tổng số 276 công ty được xem xét, các công ty tập trung nhiều nhất vào ngành công nghiệp (76 công ty), kế đến là nguyên vật liệu (46 công ty) và ngành có số công ty ít nhất là công nghệ thông tin (6 công ty), tiếp theo là chăm sóc sức khỏe ( 9 công ty) và năng lượng $(9$ công ty). Ngoài ra, kết quả nghiên cứu cũng chỉ ra tương quan SSL giữa các công ty trong ngành và ngoài ngành tương đối nhỏ và có sự khác biệt không đáng kể. Ngoại trừ ngành năng lượng, bất động sản và tài chính, hầu hết các ngành có tương quan cả trong ngành và ngoài ngành đều nhỏ hơn 0.1 . Điều này làm cho kết quả kiểm định về sự khác biệt giữa trung bình tương quan SSL của các công ty trong cùng ngành và trung bình tương quan SSL của các công ty khác ngành là không có ý nghĩa thống kê. Như vậy, nếu xét theo tiêu chí nhóm đồng nhất, hệ thống phân ngành GICS là chưa thực sự phù hợp ở TTCK Thành phố Hồ Chí Minh. Mặc dù có sự khác biệt với các nghiên cứu trước trên thế giới như Chan và cộng sự (2007), Bhojraj và cộng sự (2003), kết quả nghiên cứu có sự tương đồng với nghiên cứu của Vuong và Tran (2017) cho TTCK Thành phố Hồ Chí Minh. Như vậy, dù với số liệu cập nhật hơn, và chuẩn phân ngành GICS có sự thay đổi (bổ sung thêm ngành bất động sản), kết quả nghiên cứu cũng cho thẩy hệ thống phân ngành GICS chưa thực sự phù hợp khi áp dụng tại HOSE. Vì vậy, nghiên cứu tiếp tục thực hiện với hệ thống phân ngành TRBC. Kết quả nghiên cứu đối với hệ thống phân ngành TRBC được trình bày trong bảng sau: 


\section{Bảng 2}

Tương quan suất sinh lợi theo ngày trong ngành và ngoài ngành của hệ thống phân ngành TRBC

\begin{tabular}{|l|c|c|c|c|}
\hline \multicolumn{1}{|c|}{ Phân ngành theo TRBC } & Trong ngành & Ngoài ngành & Khác biệt & $\begin{array}{c}\text { Số Công ty } \\
\text { (Tổng = 276) }\end{array}$ \\
\hline Năng lượng (50) & 0.120 & 0.093 & 0.026 & 16 \\
\hline Nguyên vật liệu cơ bản (51) & 0.091 & 0.086 & 0.005 & 45 \\
\hline Công nghiệp (52) & 0.068 & 0.077 & -0.009 & 55 \\
\hline HTD theo chu kỳ (53) & 0.066 & 0.073 & -0.007 & 40 \\
\hline HTD không theo chu kỳ (54) & 0.054 & 0.067 & -0.013 & 32 \\
\hline Tài chính (55) & 0.168 & 0.102 & 0.066 & 55 \\
\hline Chăm sóc sức khoẻ (56) & 0.099 & 0.072 & 0.027 & 8 \\
\hline Công nghệ (57) & 0.063 & 0.081 & -0.017 & 8 \\
\hline Dịch vụ viễn thông (58) & & & & \\
\hline Dịch vụ tiện ích (59) & 0.062 & 0.066 & -0.004 & 17 \\
\hline Trung bình đơn giản & $\mathbf{0 . 0 8 8}$ & $\mathbf{0 . 0 8 0}$ & $\mathbf{0 . 0 0 7}$ & \\
\hline Trung bình có trọng số & $\mathbf{0 . 0 9 3}$ & $\mathbf{0 . 0 8 2}$ & $\mathbf{0 . 0 1 1}$ & \\
\hline t-statistic & & & 0.914 & \\
\hline p-value & & & 0.388 & \\
\hline
\end{tabular}

Nguồn: Tính toán của nhóm tác giả dựa trên Matlab 2016

Kết quả nghiên cứu cho hệ thống phân ngành TRBC chỉ ra tại TTCK Thành phố Hồ Chí Minh, trong tổng số 276 công ty được xem xét, các công ty tập trung nhiều nhất vào ngành tài chính (55 công ty) và công nghiệp (55 công ty), kế đến là nguyên vật liệu cơ bản (45 công ty) và ngành có số công ty ít nhất là dịch vụ viễn thông ( 0 công ty), tiếp theo là chăm sóc sức khỏe ( 8 công ty) và công nghệ ( 8 công ty). Ngoài ra, kết quả nghiên cứu cũng chỉ ra trung bình tương quan SSL giữa các công ty trong ngành và ngoài ngành tương đối nhỏ và có sự khác biệt không đáng kể. Tương tự như hệ thống phân ngành GICS, ngoại trừ ngành năng lượng và tài chính, hầu hết các ngành có tương quan cả trong ngành và ngoài ngành đều nhỏ hơn 0.1 . Điều này làm cho kết quả kiểm định về sự khác biệt giữa trung bình tương quan SSL của các công ty trong cùng ngành và trung bình tương quan SSL của các công ty khác ngành là không có ý nghĩa thống kê. Như vậy, nếu xét theo tiêu chí nhóm đồng nhất, hệ thống phân ngành TRBC cũng chưa thực sự phù hợp ở TTCK Thành phố Hồ Chí Minh.

Tiếp theo, nghiên cứu thực hiện phân tích với dữ liệu tuần. Các kết quả phân tích với các hệ thống phân ngành GICS và TRBC được trình bày trong Bảng 3 và Bảng 4 . Với dữ liệu SSL theo tuần, mức tương quan trung bình của các cổ phiếu cả trong ngành và ngoài ngành của cả hệ thống phân ngành GICS và TRBC đều có sự tăng nhẹ, và sự khác biệt giữa tương quan trong ngành và ngoài ngành cũng có xu hướng tăng. Tuy nhiên, ở mức ý nghĩa $5 \%$, sự khác biệt giữa tương quan SSL trung bình giữa trong ngành và ngoài ngành chưa có ý nghĩa thống kê. 
Như vậy, mặc dù kết quả có sự cải thiện so với trường hợp phân tích với dữ liệu SSL theo ngày, phân tích với dữ liệu tuần cũng chưa cho thấy tính phù hợp của hai hệ thống phân ngành GICS và TRBC đối với các cổ phiếu trên HOSE.

\section{Bảng 3}

Tương quan SSL theo tuần trong ngành và ngoài ngành của hệ thống phân ngành GICS

\begin{tabular}{|l|c|c|c|c|}
\hline & Trong ngành & Ngoài ngành & Khác biệt & Số Công ty \\
\hline Năng lượng (10) & 0.283 & 0.142 & 0.142 & 9 \\
\hline Nguyên vật liệu (15) & 0.089 & 0.089 & -0.001 & 46 \\
\hline Công nghiệp (20) & 0.110 & 0.100 & 0.010 & 76 \\
\hline HTD không thiết yếu (25) & 0.056 & 0.068 & -0.012 & 29 \\
\hline HTD thiết yếu (30) & 0.077 & 0.087 & -0.010 & 30 \\
\hline Chăm sóc sức khoẻ (35) & 0.093 & 0.074 & 0.019 & 9 \\
\hline Tài chính (40) & 0.261 & 0.132 & 0.130 & 19 \\
\hline Công nghệ thông tin (45) & 0.092 & 0.081 & 0.011 & 6 \\
\hline Dịch vụ tiện ích (55) & 0.081 & 0.082 & 0.000 & 18 \\
\hline Bất động sản (60) & 0.172 & 0.116 & 0.056 & 34 \\
\hline Trung bình đơn giản & $\mathbf{0 . 1 3 2}$ & $\mathbf{0 . 0 9 7}$ & $\mathbf{0 . 0 3 4}$ & \\
\hline Trung bình có trọng số & $\mathbf{0 . 1 1 8}$ & $\mathbf{0 . 0 9 6}$ & $\mathbf{0 . 0 2 2}$ & \\
\hline t-statistic & & & 1.922 & \\
\hline p-value & & & 0.087 & \\
\hline
\end{tabular}

Nguồn: Tính toán của nhóm tác giả dựa trên Matlab 2016

\section{Bảng 4}

Tương quan SSL theo tuần trong ngành và ngoài ngành của hệ thống phân ngành TRBC

\begin{tabular}{|l|c|c|c|c|}
\hline Phân ngành theo TRBC & Trong ngành & Ngoài ngành & Khác biệt & Số Công ty \\
\hline Năng lượng (50) & 0.154 & 0.115 & 0.039 & 16 \\
\hline Nguyên vật liệu cơ bản (51) & 0.103 & 0.099 & 0.005 & 45 \\
\hline Công nghiệp (52) & 0.089 & 0.093 & -0.004 & 55 \\
\hline HTD theo chu kỳ (53) & 0.067 & 0.078 & -0.010 & 40 \\
\hline HTD không theo chu kỳ (54) & 0.073 & 0.085 & -0.011 & 32 \\
\hline Tài chính (55) & 0.200 & 0.116 & 0.085 & 55 \\
\hline Chăm sóc sức khoẻ (56) & 0.116 & 0.076 & 0.040 & 8 \\
\hline Công nghệ (57) & 0.081 & 0.093 & -0.012 & 8 \\
\hline Dịch vụ viễn thông (58) & & & 0.000 & 0 \\
\hline Dịch vụ tiện ích (59) & 0.090 & 0.081 & 0.009 & 17 \\
\hline Trung bình đơn giản & $\mathbf{0 . 1 0 8}$ & $\mathbf{0 . 0 9 3}$ & $\mathbf{0 . 0 1 4}$ & \\
\hline Trung bình có trọng số & $\mathbf{0 . 1 1 3}$ & $\mathbf{0 . 0 9 5}$ & $\mathbf{0 . 0 1 8}$ & \\
\hline
\end{tabular}




\begin{tabular}{|l|l|l|c|c|}
\hline Phân ngành theo TRBC & Trong ngành & Ngoài ngành & Khác biệt & Số Công ty \\
\hline t-statistic & & & 1.408 & \\
\hline p-value & & & 0.197 & \\
\hline
\end{tabular}

Nguồn: Tính toán của nhóm tác giả dựa trên Matlab 2016

Từ các phân tích với dữ liệu tuần cho thấy kết quả có được cải thiện so với dữ liệu ngày, nghiên cứu tiếp tục thực hiện phân tích với dữ liệu SSL theo tháng, cho cả hai hệ thống phân ngành GICS và TRBC. Kết quả nghiên cứu được trình bày trong các Bảng 5 và Bảng 6 . Với dữ liệu SSL theo tháng, hầu hết các giá trị tăng lên so với trường hợp tính toán cho SSL theo ngày. Điều này làm cho sự khác biệt giữa trung bình tương quan SSL theo tháng giữa các công ty cùng ngành và khác ngành có sự khác biệt về mặt thống kê ở mức ý nghĩa $5 \%$. Như vậy, hệ thống phân ngành GICS và TRBC có thể phân các công ty trên HOSE thành các nhóm đồng nhất. Nói cách khác, khi xem xét dữ liệu SSL theo tháng, dựa trên tiêu chí này, hệ thống phân ngành GICS và TRBC là phù hợp để áp dụng phân ngành trên HOSE.

Như vậy, mặc dù cả hai hệ thống phân ngành GICS và TRBC chưa chứng tỏ được sự phù hợp khi phân tích dữ liệu theo ngày và theo tuần, khi phân tích dữ liệu tháng, các kết quả chỉ ra các hệ thống này là phù hợp cho HOSE. Như vậy, nghiên cứu này đã bổ sung kết quả nghiên cứu của Vuong và Tran (2017), nghĩa là các tiêu chí này chỉ không phù hợp khi phân tích dữ liệu ngắn hạn (theo ngày), trong khi sẽ phù hợp khi phân tích dữ liệu dài hạn (theo tháng). Nguyên nhân có thể giải thích cho hiện tượng này là do khi xem xét trong ngắn hạn (theo ngày), số lượng tin tức liên quan đến ngành là không nhiều, làm sự biến động giá cả của các công ty mang tính ngẫu nhiên do cung cầu nhiều hơn là do các tin tức theo ngày của từng ngành. Ngoài ra, giới hạn biên độ dao động giá trên HOSE cũng góp phần giải thích nguyên nhân cho sự khác biệt khi phân tích với SSL theo ngày và theo tháng. Giới hạn giá làm cho giá cổ phiếu khó có thể biến động mạnh, làm cho SSL cổ phiếu theo ngày bị giới hạn trong biên độ quy định, trong khi phân tích dữ liệu theo tháng, vai trò của biên độ giá hầu như không đáng kể. Điều này góp phần làm tương quan giữa các cổ phiếu khi phân tích dữ liệu theo tháng có xu hướng lớn hơn khi phân tích dữ liệu theo ngày.

\section{Bảng 5}

Tương quan SSL theo tháng trong ngành và ngoài ngành của hệ thống phân ngành GICS

\begin{tabular}{|l|c|c|c|c|}
\hline Phân ngành GICS & Trong ngành & Ngoài ngành & Khác biệt & Số Công ty \\
\hline Năng lượng (10) & 0.344 & 0.163 & 0.181 & 9 \\
\hline Nguyên vật liệu (15) & 0.105 & 0.101 & 0.004 & 46 \\
\hline Công nghiệp (20) & 0.115 & 0.110 & 0.005 & 76 \\
\hline HTD không thiết yếu (25) & 0.100 & 0.095 & 0.005 & 29 \\
\hline HTD thiết yếu (30) & 0.074 & 0.094 & -0.019 & 30 \\
\hline Chăm sóc sức khoẻ (35) & 0.189 & 0.095 & 0.094 & 9 \\
\hline Tài chính (40) & 0.285 & 0.141 & 0.144 & 19 \\
\hline Công nghệ thông tin (45) & 0.157 & 0.115 & 0.043 & 6 \\
\hline Dịch vụ tiện ích (55) & 0.150 & 0.107 & 0.043 & 18 \\
\hline
\end{tabular}




\begin{tabular}{|l|c|c|c|c|}
\hline Phân ngành GICS & Trong ngành & Ngoài ngành & Khác biệt & Số Công ty \\
\hline Bất động sản (60) & 0.175 & 0.118 & 0.057 & 34 \\
\hline Trung bình đơn giản & $\mathbf{0 . 1 6 9}$ & $\mathbf{0 . 1 1 4}$ & $\mathbf{0 . 0 5 6}$ & \\
\hline Trung bình có trọng số & $\mathbf{0 . 1 4 0}$ & $\mathbf{0 . 1 0 9}$ & $\mathbf{0 . 0 3 0}$ & \\
\hline t-statistic & & & $\mathbf{2 . 6 8 2}$ & \\
\hline p-value & & & $\mathbf{0 . 0 2 5}$ & \\
\hline
\end{tabular}

Nguồn: Tính toán của nhóm tác giả dựa trên Matlab 2016

\section{Bảng 6}

Tương quan suất sinh lợi theo tháng trong ngành và ngoài ngành của hệ thống phân ngành TRBC

\begin{tabular}{|l|c|c|c|c|}
\hline Phân ngành theo TRBC & Trong ngành & Ngoài ngành & Khác biệt & Số Công ty \\
\hline Năng lượng (50) & 0.237 & 0.144 & 0.092 & 16 \\
\hline Nguyên vật liệu cơ bản (51) & 0.106 & 0.107 & -0.001 & 45 \\
\hline Công nghiệp (52) & 0.108 & 0.106 & 0.002 & 55 \\
\hline HTD theo chu kỳ (53) & 0.087 & 0.093 & -0.006 & 40 \\
\hline HTD không theo chu kỳ (54) & 0.071 & 0.091 & -0.020 & 32 \\
\hline Tài chính (55) & 0.211 & 0.119 & 0.092 & 55 \\
\hline Chăm sóc sức khoẻ (56) & 0.229 & 0.104 & 0.125 & 8 \\
\hline Công nghệ (57) & 0.170 & 0.123 & 0.047 & 8 \\
\hline Dịch vụ viễn thông (58) & & & & 0 \\
\hline Dịch vụ tiện ích (59) & 0.170 & 0.107 & 0.062 & 17 \\
\hline Trung bình đơn giản & $\mathbf{0 . 1 5 4}$ & $\mathbf{0 . 1 1 0}$ & $\mathbf{0 . 0 3 9}$ & \\
\hline Trung bình có trọng số & $\mathbf{0 . 1 3 7}$ & $\mathbf{0 . 1 0 8}$ & $\mathbf{0 . 0 3 0}$ & \\
\hline t-statistic & & & $\mathbf{2 . 5 0 5}$ & \\
\hline p-value & & & $\mathbf{0 . 0 3 7}$ & \\
\hline
\end{tabular}

Nguồn: Tính toán của nhóm tác giả dựa trên Matlab 2016

Tóm lại, kết quả nghiên cứu đã chỉ ra các hệ thống phân ngành GICS và TRBC là phù hợp cho HOSE khi phân tích dữ liệu theo tháng. Nghiên cứu này đã góp phần bổ sung đáng kể cho nghiên cứu trước đây của (Vuong \& Tran, 2017). Như vậy, mặc dù tại HOSE, GICS và TRBC chưa phân các cổ phiếu thành các nhóm đồng nhất tốt như trên các thị trường thế giới, hai hệ thống phân ngành này vẫn có thể được sử dụng trên HOSE.

\section{Kết luận}

Nghiên cứu này đã tính toán tương quan bình quân SSL trong ngành và ngoài ngành cấp 1 cho tất cả các cổ phiếu niêm yết trên HOSE giai đoạn từ năm 2014 đến năm 2017, ứng với 2 hệ thống phân loại ngành GICS và TRBC. Trong cả hai hệ thống phân ngành, mặc dù kết quả nghiên cứu chưa cho thấy sự khác biệt có ý nghĩa về mặt thống kê giữa SSL của các công ty trong ngành và ngoài ngành đối với dữ liệu theo ngày và tuần, kết quả nghiên cứu với SSL theo tháng cho thấy sự khác biệt này có ý nghĩa thống kê. Như vậy, với dữ liệu SSL theo tháng, 
nghiên cứu cho thấy GICS và TRBC là phù hợp với HOSE theo tiêu chí phân chia các công ty niêm yết thành các nhóm đồng nhất. Kết quả nghiên cứu đã bổ sung nghiên cứu trước đây của Vuong và Tran (2017). Tóm lại, trong bối cảnh chưa nhiều nghiên cứu đánh giá sự phù hợp của các hệ thống phân ngành tại TTCK Việt Nam, nghiên cứu này đã góp phần chỉ ra sự phù hợp của hệ thống GICS và TRBC tại HOSE.

\section{LỜI CẢM ƠN}

Nghiên cứu là một phần kết quả của nghiên cứu Khoa học cấp cơ sở (Trường Đại học Sài Gòn), được thực hiện bởi nhóm tác giả Trần Thị Ngọc Quỳnh (chủ nhiệm đề tài), Lê Đình Nghi, Lê Khoa Huân, theo Hợp đồng nghiên cứu Khoa học số 875/HĐ-ĐHSG giữa Bên A là Trường Đại học Sài Gòn và Bên B là Trần Thị Ngọc Quỳnh.

\section{Tài liệu tham khảo}

Bhojraj, S., Lee, C. M. C., \& Oler, D. K. (2003). What's my line? A comparison of industry classification schemes for capital market research. $<\mathrm{i}>$ Journal of Accounting Research, $41</ \mathrm{i}>(5), 745-774$.

Chan, L. K. C., Lakonishok, J., \& Swaminathan, B. (2007). Industry return classifications and comovement. <i>Financial Analysts Journal, 63</i>(6), 56-70.

Hrazdil, K., Trottier, K., \& Zhang, R. (2013). A comparison of industry classification schemes: A large sample study. <i>Economics Letters, 118</i>(1), 77-80. doi:10.1016/j.econlet.2012.09.022

MSCI. (2016). $<\mathrm{i}>S \& P$ Dow Jones Indices and MSCI announce August 2016 creation of a real estate sector in the Global Industry Classification Standard ( GICS ® ) structure $</ \mathrm{i}\rangle$. Retrieved October $\quad 10, \quad 2019, \quad$ from https://www.msci.com/documents/10199/248121/Revisions+to+GICS+Structure+in+20 16/cfded0bf-18bc-46d9-9053-ce22ef287481

MSCI. (2018). <i $>$ The global industry classification standard $<\mathrm{i}>$. Retrieved October 20, 2019, from https://www.msci.com/gics

Phillips, R. L., \& Ormsby, R. (2016). Industry classification schemes: An analysis and review. $<\mathrm{i}>$ Journal of Business and Finance Librarianship, 21</i $>(1), \quad 1-25$. doi:10.1080/08963568.2015.1110229

Refinitiv. (2019). <i>Thomson Reuters business classification</i >. Retrieved September 20, 2019, from https://www.refinitiv.com/en/financial-data/indices/trbc-businessclassification

Vuong, Q. D. H., \& Tran, Q. T. N. (2017). Phải chăng hệ thống phân ngành toàn cầu là phù hợp cho Sở Giao dịch chứng khoán Thành phố Hồ Chí Minh [Is the global subsector system suitable for the Ho Chi Minh Stock Exchange]. <i>Tạp Chi Kinh Tế \& Phát Triển, $240</ \mathrm{i}>, 66-76$. 